Vietnam Journal of Mechanics, VAST, Vol. 42, No. 2 (2020), pp. 95-103

DOI: https://doi.org/10.15625/0866-7136/13753

\title{
DETERMINING OF THE LASER HEAT FLUX FOR THREE-DIMENSIONAL CONDUCTION MODEL BY THE SEQUENTIAL METHOD
}

\author{
Nguyen Nhut Phi Long ${ }^{1}$, Nguyen Hoai Son ${ }^{1, *}$, Nguyen Quan $^{2}$ \\ ${ }^{1}$ Ho Chi Minh City University of Technology and Education, Vietnam \\ ${ }^{2}$ Pham Van Dong University, Quang Ngai, Vietnam \\ *E-mail: sonnh@hcmute.edu.vn
}

Received: 19 April 2019 / Published online: 11 May 2020

\begin{abstract}
When performing a laser processing, one of the parameters to consider is the laser heat flux. This is a very important parameter of the processing. It is difficult to directly and correctly measure this parameter during the processing. Therefore, to estimate this parameter, a solution has been implemented. In this study, the Newton-Raphson method has been calibrated as an operational algorithm to evaluate the laser heat flux value accurately in the $3-\mathrm{D}$ conduction model. To confirm the effectiveness of the presented method, the paper has given two specific applications. It is obtained that the sequential method is a reasonable, correct, and powerful method to determine the inversely laser heat flux in the three-dimensional conduction model.
\end{abstract}

Keywords: laser processing, laser flux, 3-D heat conduction model, modified NewtonRaphson (MNR) method, sequential method.

\section{INTRODUCTION}

In recent years, the rapid development of laser processing technology has gradually replaced the traditional processing techniques. Comparing the conventional machining processing, laser processing technology is not affected by the wear of the tool and the friction during processing because of its non-contact property. Moreover, laser processing technology has many advantages, such as high energy density, quick fabrication, high precision, and low cost and non-pollution [1,2].

The absorbed energy of a laser beam known as laser heat flux is an important parameter in laser processing such as laser cladding, laser surface hardening, and laser welding. However, the direct measurement of this parameter during the process is difficult. As a result, many researchers used the inverse method to determine this parameter. For instance, Wang et al. [3] calculated this parameter on the surface. His work used the conjugate gradient method (CGM) in the surface hardening process by the laser to determine this parameter inversely. Chen and $\mathrm{Xu}$ [4] applied Laplace transform to the governing

(c) 2020 Vietnam Academy of Science and Technology 
differential equation, boundary conditions, and initial condition to evaluate absorption in the heating process for surface by laser. Yang et al. [5] applied CGM to estimate the absorbed energy of the laser beam and depth of the melt zone simultaneously. Sun et al. [6] used the method of direct sensitivity coefficient for the determining of the surface heat flux and the absorptivity of the coating surface in the surface hardening process by the laser. Nevertheless, these studies were only performed on a one-dimensional and two-dimensional model. The obtained results may be less accurate than the truly threedimensional model. Nguyen and Yang [7] used the inverse algorithm in the modified Newton-Raphson method (MNR method) to determine laser power in order to reach the required width penetration in the laser welding process. Through two examples, with every 7 iterations in each example, the speed of the method being applied is excellent. The error between the estimated width penetration and the setup width penetration in the two examples, respectively, is $0.25 \%$ and $0.2 \%$. Nguyen and Yang [8] continued to use the MNR method in estimating the absorption coefficient, which is one of the very important parameters during laser welding. The determination of this parameter depends on the temperature is the complex non-linear inverse problem. The long rod with the small diameter model is heated by the Gauss distribution laser source. The results of the two examples in this study show that the number of future time step increases, the measurement error decreases, the determined value increases, and this result with the constant relation of the future time step is less exact the linear type of the future time. Nguyen et al. [9] used the sequential method to inverse evaluation of the absorption coefficient for the spot laser welding (three-dimensional cylindrical workpiece) with Friedman's heat source model. This assessment, through two examples, includes two main processes: direct analysis and inverse analysis. Firstly, this study applied the effective heat capacity method to find temperature fields by finite element method (FEM) with the boundary conditions, and the absorption coefficient assumed as specific values. In the inverse analysis process, the unknown variables are obtained from exploring points systematically by the iterative method. With several iterations are performed, the intermediate values are substituted for the undetermined variables for the next analysis. The numerical results show that the sequential method is the inverse solution to estimate the absorption coefficient with high accuracy and stability in the spot weld by laser.

This article offers a sequential method of estimating errors for laser heat conduction flux on a three-dimensional model: In the forward problem, the setup parameter value is assumed to be known, and the temperature field in the survey domain is solved by finite element method. The solution of this process is included in calculating the sensitivity and integrating with the measured temperature from the sensor to build equations. In the reverse problem, the Modified Newton-Raphson method (the MNR method) performs several iterations to find the value of the variable to be searched. This value is used for the next time step. Thus, the value of the found variable is established in the entire time-domain.

\section{PROBLEM STATEMENT}

Considering the cubic-shaped workpiece with top surface is heated by an incident laser beam with a radius of $r_{b}$. The remaining surrounding surfaces of the sample are 
coated with insulation layers to avoid energy loss. The remaining surrounding surfaces of the sample are coated with insulation layers to avoid energy loss. Thermocouples are placed inside to record the temperature, as shown in Fig. 1.

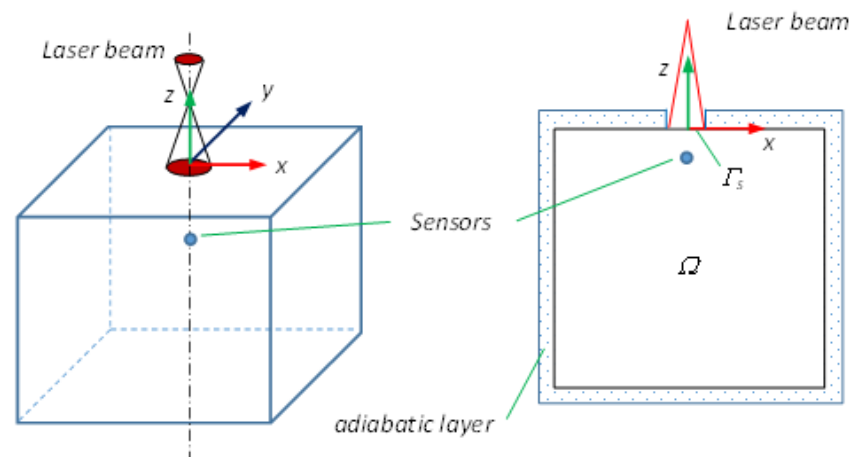

Fig. 1. Three-dimensional laser heating model

The equations of the 3-D heat conduction model take the form

$$
\begin{gathered}
\frac{\partial}{\partial x}\left[k \frac{\partial T}{\partial x}\right]+\frac{\partial}{\partial y}\left[k \frac{\partial T}{\partial y}\right]+\frac{\partial}{\partial z}\left[k \frac{\partial T}{\partial z}\right]=\rho C \frac{\partial T}{\partial t} \text { in } \Omega, 0 \leq t \leq t_{f} \\
-k \frac{\partial T}{\partial n}=I \exp \left(\frac{-\left(x^{2}+y^{2}\right)}{r_{b}^{2}}\right) \text { at } \Gamma_{s}, \\
\frac{\partial T}{\partial n}=0 \text { at otherwise surfaces, } \\
T(x, y, z, 0)=T_{0},
\end{gathered}
$$

where $k$ is a thermal conductivity $\left(\frac{\mathrm{W}}{\mathrm{m}^{\circ} \mathrm{C}}\right) ; \rho$ is a density $\left(\frac{\mathrm{kg}}{\mathrm{m}^{3}}\right) ; C$ is a heat capacity $\left(\frac{\mathrm{J}}{\mathrm{kg}^{\circ} \mathrm{C}}\right) ; T(x, y, z, t)$ is a temperature field $\left({ }^{\circ} \mathrm{C}\right), T_{0}$ is the initial temperature $\left({ }^{\circ} \mathrm{C}\right) ; \mathrm{I}$ is a laser heat flux $\left(\frac{\mathrm{W}}{\mathrm{m}^{2}}\right)$ and $r_{b}$ is an effective laser beam radius (mm); $n$ is unit normal vector.

When other input parameters, the boundary conditions and the laser heat intensity, are specified prior, the finite element method [10-12] is utilized to find the temperature field in the $\Omega$ domain. When the temperature history is measured inside the workpiece, the laser heat flux of the surface is estimated by the inverse problem.

\section{THE SEQUENTIAL METHOD}

At each time step, this method consists of four problems to be solved as follows: 


\subsection{Direct/Forward problem}

At the time $t=t_{m}$, Eqs. (1)-(4) become

$$
\begin{gathered}
\frac{\partial}{\partial x}\left[k \frac{\partial T_{m}}{\partial x}\right]+\frac{\partial}{\partial y}\left[k \frac{\partial T_{m}}{\partial y}\right]+\frac{\partial}{\partial z}\left[k \frac{\partial T_{m}}{\partial z}\right]=\rho C \frac{\partial T_{m}}{\partial t} \text { in } \Omega, t=t_{m}, \\
-k \frac{\partial T_{m}}{\partial n}=I_{m} \exp \left(\frac{-\left(x^{2}+y^{2}\right)}{r_{b}^{2}}\right) \text { at } \Gamma_{s}, \\
\frac{\partial T_{m}}{\partial n}=0 \text { at otherwise surfaces, } \\
T\left(x, y, z, t_{m-1}\right)=T_{m-1},
\end{gathered}
$$

In the forward problem, data on temperature changes at one or several points in the solder are used to calculate the inverse of this coefficient. The concept of the quantity of time in the future (the future time) is proposed and used to ensure stability and continuity during the setup process. While setting the value at time step $t=t_{m}$, the value of the variable set at the time step $t=t_{1}, t=t_{2}, \ldots, t=t_{m-1}$ has been set and some values set in the next time steps are assumed to be constant or linear in relation to the current set value. Then, the unknown surface laser heat flux is introduced as follows

$$
I_{m+r}=I_{m+r-1}=\ldots=I_{m+1}=I_{m},
$$

where $r$ is the quantity of time in the future.

And then, the solution is performed from $t=t_{m}$ to $t=t_{m+r}$ ( $r$ steps) for the direct problem shown in Eqs. (5)-(8), and the unknown absorbed energy of the laser beam is set by Eq. (9).

\subsection{Sensitivity problem}

The MNR method implements several loops to calculate the value of the variable to be searched at each time step. Because the MNR method is the gradient-based method, sensitivity analysis is needed to achieve the search step in each loop. After Eqs. (5)-(8) has been derived $\frac{\partial}{\partial I_{m}}$ for both sides

$$
\begin{gathered}
\frac{\partial}{\partial x}\left[k \frac{\partial X_{m}}{\partial x}\right]+\frac{\partial}{\partial y}\left[k \frac{\partial X_{m}}{\partial y}\right]+\frac{\partial}{\partial z}\left[k \frac{\partial X_{m}}{\partial z}\right]=\rho C \frac{\partial X_{m}}{\partial t} \text { in } \Omega, t=t_{m} \\
-k \frac{\partial X_{m}}{\partial n}=\frac{\partial}{\partial I_{m}}\left(I_{m} \exp \left(-\left(x^{2}+y^{2}\right) / r_{b}^{2}\right)\right) \text { at } \Gamma_{s}, \\
\frac{\partial X_{m}}{\partial n}=0 \text { at otherwise surfaces, } \\
X\left(x, y, z, t_{m-1}\right)=X_{m-1}=0,
\end{gathered}
$$

Eqs. (10)-(13) are the linear equations and their dependent variable is $X_{m}$, concerning independent variables $x, y, z$, and $t$. Hence, the solution of this sensitivity problem can be solved as the forward problem by the finite element method. 


\subsection{Modified Newton-Raphson (MNR) method}

In this research, the MNR method is seen in the operational algorithm [7]. After having the solution of two problems: direct and sensitivity, the inverse analysis process is satisfied when using this method. In the MNR method, the variable to be set is shown in a non-linear equation. This equation is built directly from the comparison between the calculated temperature in the forward problem and the temperature obtained from the sensor. Therefore, the response value of the sensor value $\Phi_{\text {meas }}^{j}$ and the calculated value $\Phi_{c a l}^{j}$ are predefined. The solution of this equation: the estimation of the unknown heat flux on the surface $\left(I_{m}\right)$ can be changed at each time step.

$$
\boldsymbol{\Phi}=\Phi_{\text {cal }}^{j}-\Phi_{\text {meas }}^{j}=0,
$$

where $j=m, m+1, \ldots, m+r$ is the quantity of the equations about the comparison between the measured and the calculated temperature.

It is assumed that the undetermined surface laser heat at the time step, $t=t_{m}$, is set as $\chi$. The derivative of $\boldsymbol{\Phi}$ with respect to $\chi$ can be illustrated as follows

$$
\Psi=\frac{\partial \Phi}{\partial \chi}
$$

where $\Psi$ is the sensitivity matrix $n$ summary, through the MNR method, the undetermined surface laser heat equation is as follows

$$
\chi_{k+1}=\chi_{k}+\Delta_{k}
$$

where $\Delta_{k}$ is a linear least-squares solution for a set of over-determined linear equations, and it can be derived as follows

$$
\begin{gathered}
\Delta_{k}=-\left[\boldsymbol{\Psi}^{T}\left(\chi_{k}\right) \boldsymbol{\Psi}\left(\chi_{k}\right)\right]^{-1} \boldsymbol{\Psi}^{T}\left(\chi_{k}\right) \boldsymbol{\Phi}\left(\chi_{k}\right), \\
\Rightarrow \chi_{k+1}=\chi_{k}-\left[\boldsymbol{\Psi}^{T}\left(\chi_{k}\right) \boldsymbol{\Psi}\left(\chi_{k}\right)\right]^{-1} \boldsymbol{\Psi}^{T}\left(\chi_{k}\right) \boldsymbol{\Phi}\left(\chi_{k}\right) .
\end{gathered}
$$

\subsection{The stopping criteria}

To end the loop process, the stopping criterion has been applied. In the modified Newton-Raphson method, Eqs. (14)-(17) are used to establish the unaware value $\chi$. The $\Delta_{k}$ in Eq. (17) is called a step size that runs from $\chi_{k}$ to $\chi_{k+1}$. When the stopping criterion is contented, the loop of Eq. (18) to estimate the value of $\chi$ at each time step will stop. To stop iteration, two criteria which were proposed by Frank and Wolfe [13] are selected as follows

$$
\begin{gathered}
\left\|\chi_{k+1}-\chi_{\kappa}\right\| /\left\|\chi_{k+1}\right\| \leq \delta, \\
\left\|\mathbf{J}\left(\chi_{k+1}\right)-\mathbf{J}\left(\chi_{\kappa}\right)\right\| /\left\|\mathbf{J}\left(\chi_{k+1}\right)\right\| \leq \varepsilon,
\end{gathered}
$$

where $\delta$ and $\varepsilon$ are stop values. Those are very small positive numbers.

$$
\left\|\mathbf{J}\left(\chi_{k+1}\right)\right\|=\sum_{i=1}^{r}\left[\Phi_{c}^{i}-\Phi_{\text {meas }}^{i}\right]^{2}
$$




\section{COMPUTATIONAL ALGORITHM [8]}

First, specified the quantity of time in the future $(r)$, the 3D mesh model of the domain, the time step $(\Delta t)$ in the direct problem; given a stop condition value $(\delta, \varepsilon)$ and the initial value $\left(\chi_{0}\right)$. The solution for $\chi_{k}$ at time step $t=t_{m}$ is as follows:

Step 1: Let $j=m$ and $T\left(x, y, z, t_{j-1}\right)$ is known;

Step 2: Collect the measured temperature $\Phi_{\text {meas }}^{j}$;

Step 3: Assume the initial guess $\chi_{0}$;

Step 4: Calculate the sensitivity matrix, $\Psi$, by Eqs. (10-(13));

Step 5: Solve the direct problem by Eqs. (5)-(8), and then compute the calculated temperature $\Phi_{\text {cal }}^{j}$;

Step 6: Construct $\Phi$ by $\Phi_{\text {meas }}^{j}$ and $\Phi_{\text {cal }}^{j}$;

Step 7: Knowing $\boldsymbol{\Psi}$ and $\boldsymbol{\Phi}$, determine the step size $\Delta_{k}$ by Eq. (17);

Step 8: Knowing $\Delta_{k}$ and $\chi_{k}$, calculate $\chi_{k+1}$ through Eq. (18);

Step 9: Terminate the iteration if the stopping criterion (Eqs. (19)-(20)) is satisfied. Otherwise, return to Step 5;

Step 10: Stop the process if the final time step is attached. Otherwise, let $j=m+1$ return to Step 2.

\section{RESULTS AND DISCUSSIONS}

To demonstrate the proposed method, two applications of the different heat flux are performed and the cubic-shaped substrate with the dimension of $20 \times 20 \times 20(\mathrm{~mm})$ is considered. The workpiece is made by Al1100 which thermal properties is as following: $\mathrm{C}=904\left(\frac{\mathrm{J}}{\mathrm{kg}^{\circ} \mathrm{C}}\right), k=222\left(\frac{\mathrm{W}}{\mathrm{m}^{\circ} \mathrm{C}}\right)$ and $\rho=2710\left(\frac{\mathrm{kg}}{\mathrm{m}^{3}}\right)$. The thermocouple is located at $T_{K 1}(0,0,-0.001)$.

In addition, the measured temperature is generated from Eqs. (1-(4)) when the input parameters are preselected and it is presumed to have measurement errors. In other work, the random errors of measurement are added to the exact temperature. It can be achieved in the following equation.

$$
T^{\text {meas }}=T^{\text {exact }}+\lambda \sigma,
$$

where $T^{\text {meas }}$ known as the temperature data is measured, $T^{\text {exact }}$ known as the exact temperature, $\lambda$ known as the random number (within $-2.576 \leq \lambda \leq 2.756$ ) are used from the IMSL subroutine DRNNOR [14] which presents the $99 \%$ confidence, and $\sigma$ known as the standard deviation.

The fine mesh is in the area near the laser with a size of about $\Delta x \approx 2.10^{-5}(\mathrm{~mm})$ and the coarse mesh is in the other area with a size of about $\Delta x \approx 2 \cdot 10^{-3}(\mathrm{~mm})$ (as shown in Fig. 2). As well, the time increment is 0.02 (s).

The relative average errors $\mu$ (the RA errors $\mu$ ) to inspect the deviation of the estimated results from the exact solution obtained from the direct problem shown in Eq. (23)

$$
\mu=\frac{1}{N_{t}} \sum_{i=1}^{N_{t}}\left|\frac{f-\hat{f}}{\hat{f}}\right|,
$$




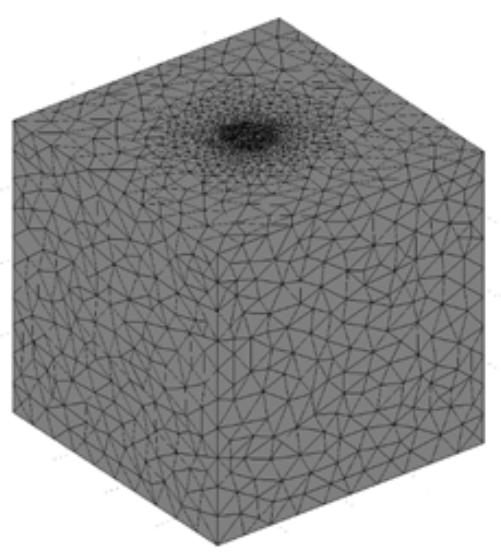

Fig. 2. The 3D mesh model of the $\Omega$ domain

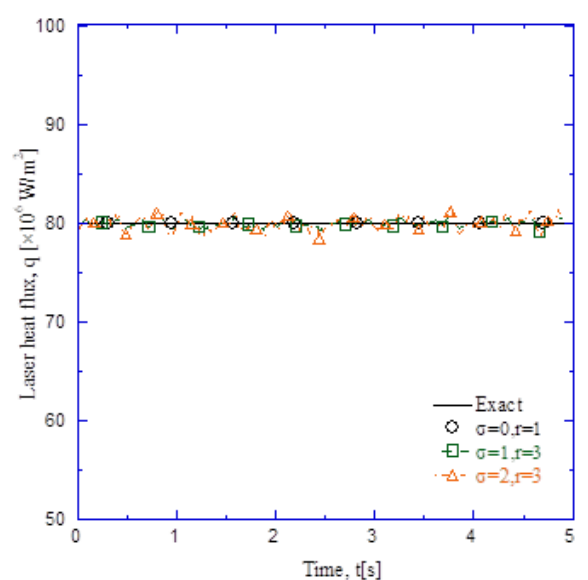

Fig. 3. The results is estimated in Application 1

where $f$ is the inverse determining results with measurement errors, $\hat{f}$ is the result of exact solution and, $N_{t}$ is the number of the temporal step. This equation shows that the value of $\mu$ is small, the setting result is still accurate.

Two applications are performed following as:

\subsection{Application 1}

The sample is initially at a uniform temperature $T_{0}=27\left({ }^{\circ} \mathrm{C}\right)$, and then is suddenly heated by a laser beam with its effective radius of $r_{b}=0.0014(\mathrm{~mm})$ and constant heat flux of $q=80 \times 10^{6}\left(\frac{\mathrm{W}}{\mathrm{m}^{2}}\right)$ at the center of the substrate top surface. Fig. 3 shows the results of the laser heat flux is estimated in Application 1. Thus, with $r=1$ and $\sigma=0$, the heat flux values are determined to be approximate with the correct solution.

For the case of the measurement errors, the estimated results with large error diverge from the accurate solution. Tab. 1 shows the RA errors $\mu$ of the inverse determined results when the errors of measurement are involved.

As shown in Tab. 1, the RA errors decrease

Table 1. The RA errors in Application 1 with the quantity of time in the future $r=3$

\begin{tabular}{cc}
\hline Cases & Relative average error \\
\hline$\sigma=1$ & 0.0031 \\
$\sigma=2$ & 0.0061 \\
\hline
\end{tabular}
with the reduction of the errors of measurement. Particularly, the RA errors in Tab. 1 decrease from 0.0031 to 0.0061 , about $49 \%$ when the errors of measurement reduce from $\sigma$ $=1$ to $\sigma=2$. In the case of $\sigma=2$, the RA error is 0.0061 and the value is small.

\subsection{Application 2}

In this application, the time variation of laser heat flux is assumed as follows

$$
q(t)=1.5 \times 10^{6} \exp (t / 2)(\sin (t)+\cos (t)+t) .
$$

Fig. 4 shows the results of the absorbed energy of the laser beam is estimated in Application 2. 


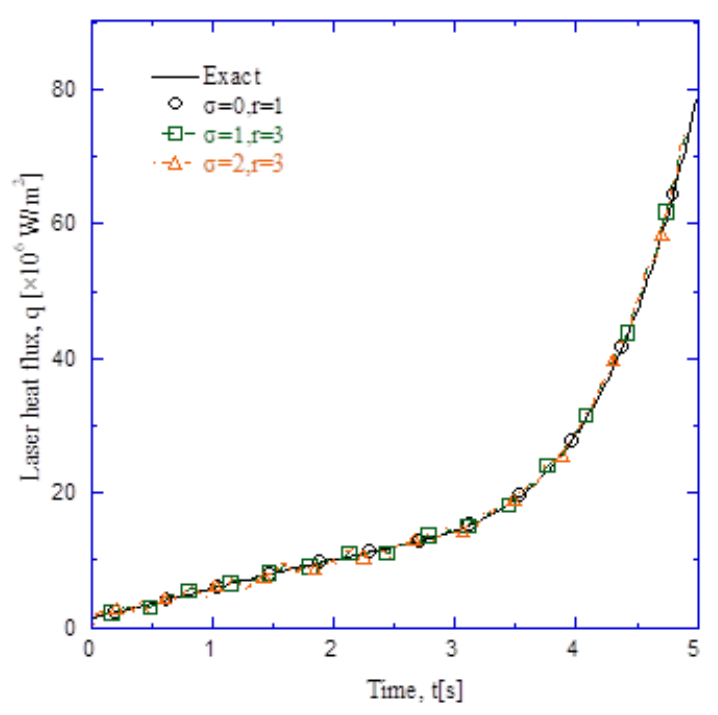

Fig. 4. The results is estimated in Application 2

Once again, Fig. 4 shows that the heat flux values are determined still to reach approximate values in the case of measurement freeerror and, even if $\sigma=1, \sigma=2$, the estimated values still approximate with the exact solution. The RA errors of the estimated results in Application 2 are shown in Tab. 2.

In the case of $\sigma=2$, the RA error is 0.0063 .

These values are still small. In all cases, the average number of iterations is about 3 at each time step. The total number of iterations is 750 . In other words, the speed of convergence of the proposed method is fast.

\section{CONCLUSIONS}

In this paper, the laser heat flux for the 3D model is estimated through the use of the sequential method. With 10 steps in the algorithm, corresponding to each time step, this method has solved four problems, including the direct/forward problem, the sensitivity problem, the Modified Newton-Raphson method, the stopping criteria. Two applications have been raised to confirm the proposed method. The results of the applications show that in the case of the measurement free-errors, the evaluated values of the absorbed energy of the laser beam has approximated with the exact solution. And in the remaining case stated, the relative average errors are also very small.

\section{REFERENCES}

[1] S. Katayama. Handbook of laser welding technologies. Elsevier, (2013). 
[2] W. M. Steen and J. Mazumder. Laser material processing. Springer Science \& Business Media, (2010).

[3] J.-T. Wang, C.-I. Weng, J.-G. Chang, and C.-C. Hwang. The influence of temperature and surface conditions on surface absorptivity in laser surface treatment. Journal of Applied Physics, 87, (7), (2000), pp. 3245-3253. https://doi.org/10.1063/1.372331.

[4] H.-T. Chen and X.-Y. Wu. Estimation of surface absorptivity in laser surface heating process with experimental data. Journal of Physics D: Applied Physics, 39, (6), (2006). https://doi.org/10.1088/0022-3727/39/6/020.

[5] Y.-C. Yang, T.-S. Wu, and E.-J. Wei. Modelling of simultaneous estimating the laser heat flux and melted depth during laser processing by inverse methodology. International Communications in Heat and Mass Transfer, 34, (4), (2007), pp. 440-447. https://doi.org/10.1016/j.icheatmasstransfer.2007.01.010.

[6] Y.-S. Sun, C.-I. Weng, T.-C. Chen, and W.-L. Li. Estimation of surface absorptivity and surface temperature in laser surface hardening process. Japanese Journal of Applied Physics, 35, (6R), (1996). https://doi.org/10.1143/jjap.35.3658.

[7] Q. Nguyen and C.-Y. Yang. Inverse determination of laser power on laser welding with a given width penetration by a modified Newton-Raphson method. International Communications in Heat and Mass Transfer, 65, (2015), pp. 15-21. https://doi.org/10.1016/j.icheatmasstransfer.2015.04.003.

[8] Q. Nguyen and C.-Y. Yang. A modified Newton-Raphson method to estimate the temperature-dependent absorption coefficient in laser welding process. International Journal of Heat and Mass Transfer, 102, (2016), pp. 1222-1229. https://doi.org/10.1016/j.ijheatmasstransfer.2016.07.034.

[9] L. N. P. Nguyen, Q. Nguyen, S. H. Nguyen, and T. T. Le. A sequential method in inverse estimation of the absorption coefficient for the spot laser welding process. In The 9th International Conference on Computational Methods (ICCM2018), (2018), https:/ /www.sci-entech.com/ICCM2018/PDFs/3464-11548-1-PB.pdf.

[10] B. Conahan, H. A. Luther, and J. O. Wilkes. Applied numerical methods. John Wiley and Sons, New York, (1969).

[11] M. N. Nguyen, T. T. Truong, and T. Q. Bui. An enhanced nodal gradient finite element for non-linear heat transfer analysis. Vietnam Journal of Mechanics, 41, (2), (2019), pp. 127-139. https://doi.org/10.15625/0866-7136/12977.

[12] B. X. Thang, N. X. Hung, and N. T. Phong. On stabilization of the node-based smoothed finite element method for free vibration problems. Vietnam Journal of Mechanics, 32, (3), (2010), pp. 167-181. https://doi.org/10.15625/0866-7136/32/3/303.

[13] J. V. Beck, B. Blackwell, and C. R. S. Clair Jr. Inverse heat conduction: Ill-posed problems. James Beck, (1985).

[14] IMSL. Library edition 10.0, User's manual: Math library version 1.0. Houston, Tex, (1987). 